\title{
La excelencia académica de mujeres con discapacidad visual en la Universidad: aportes de la teoría fundamentada
}

The Academic Excellence ofWomen with Visual Disability in the University: Contributions from GroundedTheory

Excelência académica das mulheres com deficiência visual na Universidade: contribuição a partir de uma teoria fundamentada

\author{
María Dolores Yerga-Míguez* iD orcid.org/0000-0002-1550-133X \\ Marcela Ramírez-Morera** iDorcid.org/0000-0002-5498-5656 \\ Rosa María Díaz-Jiménez*** iDorcid.org/0000-0003-3289-6393
}

\section{Para citar este artículo}

Yerga-Míguez, M. Ramírez-Morera, M. y Díaz-Jiménez, R. (2021). La excelencia académica de mujeres con discapacidad visual en la Universidad: aportes de la teoría fundamentada. Revista Colombiana de Educación, 1(83), 1-21. https://doi.org/10.17227/rce.num83-10797.

Fecha de recepción: 28/11/2019

Fecha de evaluación: 06/08/2020

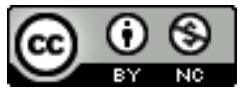

*Graduada en Trabajo Social, Máster en Género e Igualdad. Doctoranda en Ciencias Sociales. Colaboradora Honoraria del Departamento de Trabajo Social y Servicios Sociales de la Universidad Pablo de Olavide. Correo: mdyermig@alu.upo.es **Doctora en Educación y Docente en la Escuela de Trabajo Social en la Universidad de Costa Rica.

Correo: marcela.ramirez@ucr.ac.cr

***Doctora en Ciencias Sociales. Profesora Titular de Universidad del Departamento de Trabajo Social y Servicios Sociales. Universidad Pablo de Olavide. Correo: rdiajim@upo.es 


\section{Resumen}

Palabras clave: accesibilidad;

excelencia en la educación;

estudios sobre la mujer;

adaptaciones académicas;

estudiantes universitarios
En este artículo de investigación se realiza una aproximación a la vida académica de mujeres con discapacidad visual con el objetivo de analizar sus historias formativas y los factores que contribuyen a la excelencia académica. De esta forma, se presenta un estudio comparado de casos de dos mujeres con discapacidad visual matriculadas en universidades de Costa Rica y España, a partir de una hermenéutica cualitativa y una adaptación de la teoría fundamentada de diseño constructivista. Los datos se recolectaron con entrevistas semiestructuradas y se analizaron con el método comparativo constante con apoyo del software Atlas-ti v. 8. Las participantes fueron ocho, cuatro unidades de observación por cada caso y país: la mujer con discapacidad visual, un familiar, un docente y un compañero/a de clase. El marco de análisis incluyó cuatro dimensiones: personal, entorno familiar, académico y social. Como resultados destacan que los factores exógenos vinculados con la excelencia académica sitúan a la familia, el grupo de iguales (compañeros/as) y el equipo docente como apoyos permanentes a las mujeres con discapacidad visual en sus trayectorias académicas. Sin embargo, la familia influye de manera ambivalente, como apoyo y barrera, donde emerge un déficit de concienciación, especialmente entre profesorado y alumnado. Los factores endógenos que favorecen la excelencia académica se relacionan con el esfuerzo, desempeño y actitud individual, cuyo eje principal queda constituido por las características resilientes desarrolladas. Destaca como categoría emergente la violencia presente en el ámbito académico en diversas manifestaciones.
Keywords: accessibility; excellence in education; women's studies; academic accommodations; college students

\section{Abstract}

An approach to the academic life of women with visual disabilities is made to analyze academic stories that contribute to academic excellence. A comparative study of cases of two women with visual disability enrolled in universities in Costa Rica and Spain is presented based on a qualitative hermeneutic and an adaptation of the grounded theory of constructivist design. Data were collected through semi-structured interviews and analyzed with the constant comparative method supported by Atlas-ti v. 8. The participating subjects were eight, four observation units for each case and country: the woman with visual disabilities, a family member, a teacher, and a classmate. The analysis framework included four dimensions: personal, family, academic and social environment. The results highlight that exogenous factor linked to academic excellence place the family, the peer group (classmates), and the teaching team as permanent support for women with visual disability in their academic careers. However, the family has an ambivalent influence as support and barrier and a deficit of awareness emerges, especially among teachers and students. The endogenous factors that favour academic excellence are related to individual effort, performance and attitude, whose main axis is constituted by the resilient characteristics developed. The violence present in the academic environment in various forms stands out as an emerging category.

\section{Resumo}

Neste artigo de pesquisa é feita uma abordagem à vida académica das mulheres com deficiência visual com 0 objetivo de analisar as suas histórias formativas e os fatores que contribuem para a excelência académica. É apresentado um estudo comparativo do caso de duas mulheres com deficiência visual inscritas em universidades da Costa Rica e Espanha, com base numa hermenêutica qualitativa e na adaptação da teoria fundamentada de desenho construtivista. Os dados foram recolhidos através de entrevistas semiestruturadas e analisados utilizando o método comparativo constante com 0 apoio do software Atlas-ti v. 8. Havia oito participantes, quatro unidades de observação para cada caso e país: a mulher com deficiência visual, um membro da família, um professor e um colega de sala de aula. 0 quadro de análise incluiu quatro dimensões: ambiente pessoal, familiar, académico e social. Os resultados mostram que os fatores exógenos ligados à excelência académica colocam a família, o grupo de pares e o pessoal docente como apoios permanentes para as mulheres com deficiência visual nas suas trajetórias académicas. No entanto, a família tem uma influência ambivalente, tanto como apoio como como barreira, e emerge uma falta de consciência, especialmente entre professores e estudantes. Os fatores endógenos que favorecem a excelência académica estão relacionados com 0 esforço, 0 desempenho e a atitude individual, cujo eixo principal é constituído pelas características resilientes desenvolvidas. A violência, presente no meio académico em várias manifestações, destaca-se como uma categoria emergente. 


\section{Introducción}

Históricamente, las realidades de las mujeres con discapacidad visual (MDV) se han visto impregnadas por situaciones de vida que han generado condiciones de discriminación y exclusión social de una manera interseccional, entendiendo esta mirada como la interrelación de desigualdades sociales que convergen en sus vidas (Corona y Díaz-Jiménez, 2016).

El objeto de esta investigación supone analizar historias académicas que contribuyen a la excelencia universitaria de MDV, entendiendo que llegar a la universidad ya es un valor. Poner el foco aquí tiene la finalidad de profundizar sobre la excelencia académica e identificar los factores relacionados con ella en los estudios superiores de las MDV.

En cuanto al estado de la cuestión, Novo et al. (2015), Trotta et al. (2015) y Samudio y Edda (2016) analizan el recorrido histórico de las mujeres con discapacidad en la educación superior. Asimismo, estudian los factores académicos desde la perspectiva cuantitativa destacando niveles diferenciales predictivos, percepciones, impactos y efectos negativos relacionados con el éxito académico (Heiman, 2006; Reed et al., 2009; Karimi, 2012; Polo y López, 2015). En los métodos de accesibilidad en educación se destacan las técnicas de lectura fácil (Barbosa, 2019) y aulas de tecnología (Molina, 2019). Por su parte, Rodríguez y Valenzuela (2019) estudiaron el acceso y permanencia de estudiantes en las universidades, pero no concretaron la discapacidad de tipo visual. En lo referido a la percepción del estudiantado universitario con discapacidad visual acerca de su desarrollo en competencias digitales es estudiada por Aquino et al. (2016), y refieren poco adelanto en las mismas. Además, Díaz y Serrato (2016) refieren que los estudios más comunes en el ámbito de la discapacidad se enfocan hacia las barreras de la inclusión educativa. Las autoras afirman que la literatura académica se desarrolla en la línea de analizar el acceso, el ámbito actitudinal, el estudio los servicios y programas de apoyo dentro de las universidades y en la actitud del profesorado.

La percepción del estudiantado universitario con discapacidad visual en relación con el desarrollo en competencias digitales es estudiada por Aquino et al. (2016), y refieren poco adelanto en las mismas. Además, Díaz y Serrato (2016) exponen que los estudios más comunes en el ámbito de la discapacidad se enfocan en las barreras de la inclusión educativa. Afirman que la literatura académica se desarrolla en la línea de analizar el acceso, el ámbito actitudinal, el estudio de los servicios y programas de apoyo dentro de las universidades y en la actitud del profesorado sin analizar las cuestiones específicas de las mujeres.

Si el análisis de la excelencia se realiza a través de una evaluación con medidas cuantitativas específicas, Oravec (2017) alerta de que pueden ser muy manipulables, ya que la evaluación es por sí misma subjetiva (Lamont, citado en O'Connor y O'Hagan, 2016). Con respecto a los rasgos propiciadores del éxito académico, Cabrera et al. (2006) expresan la necesaria presencia de rasgos como la persistencia, motivación, capacidad de esfuerzo y, para superar las dificultades, metas claras a largo plazo y constancia.

Esta investigación parte de la concepción de que la excelencia académica debe ser separada de elementos cuantitativos, pues es la persona quien construye la excelencia académica y no la competitividad social o un valor numérico. La excelencia académica puede suponer un concepto efímero, intrínsecamente relacionado con las historias académicas de las mujeres protagonistas, así lo expresan Moriña y Melero (2016) cuando se refieren al éxito académico 
La excelencia académica de mujeres con discapacidad visual en la Universidad: aportes de la teoría fundamentada | María Dolores Yerga-Míguez,

Marcela Ramírez-Morera y Rosa María Díaz-Jiménez

como el acceso a la universidad, la permanencia y la finalización de los estudios universitarios. Así pues, la trayectoria personal supondrá un mayor valor.

Igualmente, se parte de la premisa de que existen factores de influencia en el entorno de las mujeres con discapacidad que contribuyen a la excelencia académica. Concretamente, se pone en relevancia el papel significativo de la familia, como apoyo en la carrera vital. Son diversos los estudios (Rea et al., 2014; Villavicencio y López, 2017; Araújo et al. 2016) y experiencias que destacan el valor de la persona de apoyo, la madre quien en la mayoría de los casos supone un eje central en la vida de las personas con discapacidad. Otra realidad con la que se convive es la académica, con las relaciones entre las personas docentes y los grupos de pares, que en muchas ocasiones supone un gran apoyo para la MDV y de quien nos interesa conocer su forma de ver la realidad, cómo se adapta la clase y cuáles son los apoyos educativos recibidos.

El universo de contrastación se sitúa en dos universidades públicas de Costa Rica y España. En ambos países existen políticas sociales vinculadas al ámbito de la discapacidad, cuyo objetivo principal es el reconocimiento de la educación como un derecho. En el contexto español, la Ley General de Derechos de las Personas con Discapacidad y de su Inclusión Social (Ley 1/2013) específicamente incorpora aspectos para la inclusión universitaria en el artículo 20. Por su parte, Costa Rica cuenta con la Ley de Igualdad de Oportunidades para las Personas con Discapacidad (7600), que centra los derechos educativos específicamente en su artículo del 14 al 22. Ambas naciones se acogen a la Convención Internacional de Naciones Unidas sobre los Derechos de las Personas con Discapacidad (CDPD, 2006), la cual sienta las bases legislativas.

A pesar de las políticas orientadas a la inclusión, la situación de las alumnas con discapacidad sigue mostrando realidades desiguales respecto al resto de estudiantes. En ambos países se presenta lo que Rodríguez y Cano (2015) denominan "efecto embudo" en el paso de la secundaria al bachillerato y en el trascurso de la educación universitaria. En España, el cuarto informe "Universidad y discapacidad" (Fundación Universia, 2019), indica que el $1,5 \%$ de la población universitaria española tiene discapacidad, el 1,8\% realiza estudios de grado, el 1,2\% accede al posgrado y solo el 0,7\% estudia el doctorado.

En relación con el contexto costarricense, en el informe del Estado de la educación costarricense (2017) entre el 2006 y el 2016 la matrícula total de las cinco universidades públicas aumentó en casi 30000 estudiantes con un promedio anual de 3,5\%, aunque existe un incremento del $30,2 \%$ en las matrículas. No obstante, esta población solo accede a la educación superior en un $17 \%$ en comparación con las personas sin discapacidad que ingresa en un $25 \%$ (Instituto Nacional de Estadísticas y Censos [INEC] y Consejo Nacional de Personas con Discapacidad [Conapdis], 2019).

Este trabajo aplica una metodología cualitativa analizando los discursos de ocho actores mediante entrevistas en profundidad semiestructuradas y se analizan a través de la adaptación de la teoría fundamentada (Glaser y Strauss, 1967) con el apoyo de Atlas-ti 8. El origen de los datos parte de dos casos seleccionados de la tesis doctoral y el trabajo de fin de grado de dos de las autoras.

En cuanto a los resultados, se clasifican en factores exógenos y endógenos. En los primeros se analiza el ámbito familiar y social e institucional. Por otro lado, en los endógenos se concretan las características personales de las MDV. Por último, y como categoría emergente, se presentan distintas situaciones de violencia. 


\section{Metodología}

Se desarrolla una metodología basada en la investigación cualitativa (Taylor y Bogdan, 1987) y se estructura, según lo propuesto por Levitt et al. (2018), para mostrar la integridad metodológica revisando los criterios que proponen para informes de investigación cualitativa. El proceso seguido para establecer la fidelidad al tema estudiado se concreta en el acercamiento al diálogo discursivo de las personas entrevistadas con una adaptación de la teoría fundamentada (Glaser y Strauss, 1967). La recopilación de datos se realiza a través de entrevistas semiestructuradas. Además, se recurre a elementos transductivos (Ibáñez, 1985). La estrategia de análisis se apoya en el estudio comparado de casos (Yin, 1994; Coller, 2005), adecuadas en metodología cualitativa (Stake, 1995). Las entrevistas permiten analizar los discursos con un proceso dialéctico basado en la interacción de los objetivos y las preguntas de investigación (tabla 1).

Tabla 1.

Marco de análisis para abordar la excelencia en MDV universitarias

\begin{tabular}{|c|c|c|c|c|}
\hline Objeto de análisis & $\begin{array}{l}\text { Objetivo } \\
\text { general }\end{array}$ & $\begin{array}{l}\text { Objetivos } \\
\text { específicos }\end{array}$ & $\begin{array}{l}\text { Preguntas de } \\
\text { investigación }\end{array}$ & $\begin{array}{l}\text { Diálogos } \\
\text { discursivos }\end{array}$ \\
\hline $\begin{array}{l}\text { Participantes: } \\
2 \text { mujeres con DV } \\
2 \text { familiares } \\
2 \text { docentes } \\
2 \text { compañeros/as } \\
\text { Instrumento } \\
\text { recogida de datos } \\
\text { Entrevista } \\
\text { semiestructurada }\end{array}$ & $\begin{array}{l}\text { Analizar historias } \\
\text { académicas que } \\
\text { contribuyen a la } \\
\text { excelencia } \\
\text { universitaria en } \\
\text { MDV, a través de } \\
\text { un estudio de } \\
\text { casos entre } \\
\text { Costa Rica y } \\
\text { España. }\end{array}$ & $\begin{array}{l}\text { Identificar } \\
\text { factores de } \\
\text { influencia en } \\
\text { itinerarios } \\
\text { académicos de } \\
\text { MDV en estudios } \\
\text { superiores. } \\
\text { Conocer apoyos } \\
\text { recibidos por } \\
\text { estudiantes } \\
\text { universitarias } \\
\text { con } \\
\text { discapacidad } \\
\text { visual. }\end{array}$ & $\begin{array}{l}\text { ¿Cómo ha influido } \\
\text { el entorno familiar, } \\
\text { académico y social } \\
\text { de las MDV } \\
\text { estudiadas para su } \\
\text { consecución de la } \\
\text { excelencia } \\
\text { académica? } \\
\text { ¿Qué apoyos han } \\
\text { recibido las MDV en } \\
\text { su desarrollo } \\
\text { universitario? }\end{array}$ & $\begin{array}{ll}\text { Itinerarios } \\
\text { académicos. } \\
\text { Relaciones } \\
\text { familiares. } \\
\text { - Participación } \\
\text { universitaria. } \\
\text { Interacción } \\
\text { con docentes } \\
\text { e iguales. }\end{array}$ \\
\hline
\end{tabular}

Fuente: elaboración propia. 
La excelencia académica de mujeres con discapacidad visual en la Universidad: aportes de la teoría fundamentada | María Dolores Yerga-Míguez, Marcela Ramírez-Morera y Rosa María Díaz-Jiménez

\section{Instrumentos}

Los instrumentos y herramientas empleadas se eligen en función a la utilidad que aportan a la hermenéutica interpretativa necesaria para el análisis. El instrumento empleado para la recogida de datos es una entrevista semiestructurada utilizada en dos investigaciones anteriores vinculadas con la temática, promovidas por las autoras, aplicadas en cada una de las unidades de observación (MDV, familiar, docente y compañero/a). Para la elección de los casos, se utiliza el muestreo intencional teórico (Vallés, 1999), ya que, según Bonilla-Castro y Sehk (2005), permite seleccionar los casos característicos de la población limitando la muestra a estos casos. Se realiza el estudio a dos MDV, dos casos que han desarrollado un proceso académico en dos universidades públicas de España y Costa Rica. Cada caso comporta cuatro entrevistas.

Se establece una estrategia de análisis apoyada en el estudio comparado de casos (Yin, 1994; Coller, 2005), adecuadas en metodología cualitativa (Stake, 1995). Para el análisis de las ocho entrevistas que conforman los dos casos se genera una unidad hermenéutica (UHA) apoyada en el software Atlas-ti v. 8. Se parte de la hermenéutica interpretativa y se codifica la UHA a través de la codificación abierta, codificación axial y la codificación selectiva (Strauss y Corbin, 2016).

El análisis de los discursos aborda la familia de origen, por la influencia potencial en la mujer, el ámbito académico con la experiencia de docentes de las MDV y los grupos de pares que han compartido el proceso académico universitario. Las dimensiones analizadas en los discursos del resto de unidades de observación (familiares, docentes e iguales) incluye la relación de la persona con la MDV, la percepción de la discapacidad, el ámbito universitario y el desarrollo de la MDV, al igual que la percepción sobre el comportamiento en general del profesorado y la institución. También, se aborda específicamente la percepción de la excelencia académica por parte de estos actores.

El desarrollo de las entrevistas se realiza de forma presencial por parte de las investigadoras, en los respectivos lugares de trabajo de las personas entrevistadas. La duración media de las entrevistas es de una hora y media. Todas las entrevistas fueron grabadas y transcritas por las investigadoras. En relación con las consideraciones éticas a cada persona se le brinda el consentimiento informado, el cual explicita la participación voluntaria, el resguardo de su confidencialidad y la utilización de la información para fines académicos.

\section{Caracterización de las personas participantes}

La mujer con discapacidad visual española (C1.P1) tiene 31 años, es soltera, reside en zona urbana. Graduada en Trabajo Social en el 2014, es técnica en Integración Social, especialista en mediación y máster en Género e Igualdad. Actualmente es doctoranda en Ciencias Sociales en una universidad pública española, a la vez que imparte docencia universitaria. Su carrera académica ha sido desarrollada en instituciones privadas-concertadas y públicas. Por su parte, la mujer costarricense (C2.P2) tiene 33 años, está casada y tiene una hija. Es procedente de zona rural, graduada en Trabajo Social en una institución pública en 2011. Ha sido docente universitaria, aunque actualmente estudia una maestría en una universidad pública de Costa Rica. Desarrolló su carrera académica en instituciones públicas, en zona rural y urbana. Ambas mujeres tienen una discapacidad visual desarrollada por la misma enfermedad congénita. La tabla 2 describe la codificación empleada para la identificación de las personas entrevistas.

Número 83, Tercer cuatrimestre de 2021 (septiembre-diciembre) || e-ISSN: 2323-0134 || 6 
La excelencia académica de mujeres con discapacidad visual en la Universidad: aportes de la teoría fundamentada | María Dolores Yerga-Míguez, Marcela Ramírez-Morera y Rosa María Díaz-Jiménez

Tabla 2.

Identificación de las unidades de observación por caso

\begin{tabular}{lll}
\hline $\begin{array}{l}\text { Unidades de } \\
\text { observación }\end{array}$ & (Caso España) & (Caso Costa Rica) \\
\hline Mujer DV & C1.P1 & C2.P2 \\
Familiar & C1.F1 & C2.F2 \\
Iguales & C1.C1 & C2.C2 \\
Docente & C1.D1 & C2.D2 \\
\hline
\end{tabular}

Fuente: elaboración propia.

La inclusión de las personas representantes del ámbito familiar, institucional y social ha sido elección propia de las mujeres. En el caso 1, su ámbito familiar lo representa la madre (C1.F1), de 59 años, divorciada, de zona urbana y de profesión técnica administrativa. La mujer costarricense eligió a su pareja (C2.F2) como persona relevante para explicar su trayectoria académica, de 33 años, residente en zona urbana y con estudios de secundaria.

Cuando se habla de grupo de pares o grupo de iguales, se incluyen en esta categoría a personas que han compartido aula y rol de estudiante con las mujeres en la universidad y que brindan un testimonio cercano de esta etapa. La estudiante española (compañera, igual) es una mujer (C1.C1) de 25 años y soltera. Estudió el grado de trabajo social y con empleo en una entidad pública. Por otra parte, el caso costarricense es representado por una compañera (C2.C2) de 32 años, soltera, trabajadora social empleada en una institución pública.

El discurso del ámbito institucional se nutre mediante dos docentes que fueron de especial significado en la trayectoria de las estudiantes analizadas, por su fomento de la inclusión en el aula y el apoyo dentro y fuera de ella. El docente español (C1.D1) tiene 41 años, casado, trabajador social y doctor en ciencias sociales. Por su parte, el docente costarricense (C2.D2), de 55 años, está divorciado y es trabajador social.

\section{Proceso de codificación}

Para el análisis de la información se plantea un proceso de codificación, que incluye tres momentos: codificación abierta, axial y selectiva (Strauss y Corbin, 2016). Una vez transcritas las entrevistas se inicia la codificación abierta a la luz del discurso presentado en cada una de ellas. Se realiza, en primer lugar, una lectura exhaustiva y una primera aproximación de códigos donde surgieron 484 códigos abiertos que fueron depurados para eliminar códigos con significado semejante.

En el proceso de codificación axial, los códigos se reagruparon en un total de seis familias; datos sociodemográficos, factores exógenos, factores endógenos, influencias del contexto (accesibilidad o barreras), conceptos generales (emergentes), junto con una clasificación final de códigos en vivo que refuerza a los anteriores.

Es necesario establecer el significado de las familias más significativas a nivel conceptual. Existen diversas formas de entender los factores endógenos, según lo propuesto por Moreira (2009) aglutinan el perfil demográfico, las condiciones familiares, los antecedentes 
académicos y la disposición hacia una disciplina. Además, se concreta como endógenos todos los elementos que influyen desde la experiencia de la mujer, así las propias características, sus sentimientos y el diagnóstico médico forman parte de estas. Los factores exógenos son descritos por Moreira (2009) como los concernientes a las características del docente, estrategias metodológicas, relaciones socio-dinámicas y el escenario institucional. En esta investigación se asume la presencia del ámbito familiar y escolar, donde se incluye el grupo de iguales y la experiencia con el docente, pero se precisa la creación de una categoría concreta sobre la influencia del contexto. Por la especial significancia en la experiencia de las MDV estudiadas, se crean las categorías de accesibilidad y barreras, junto a conceptos generales que surgieron de forma emergente (figura 1).

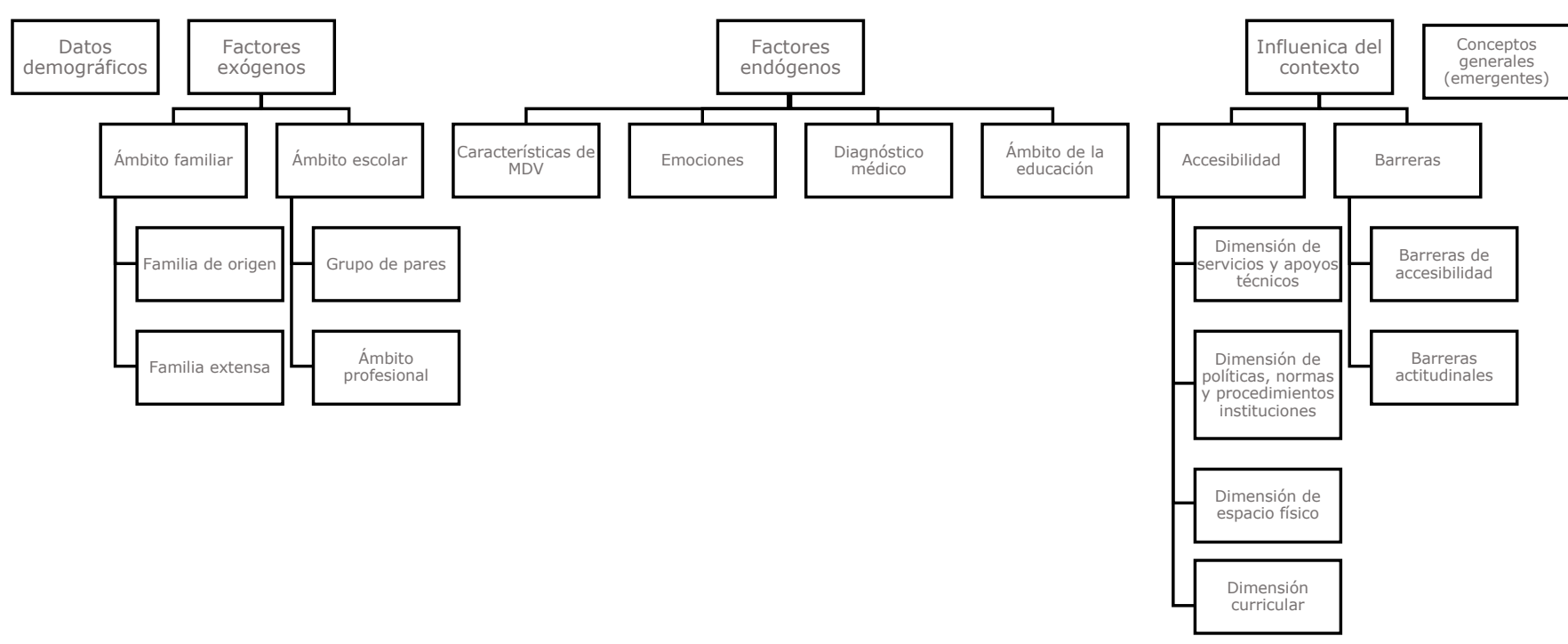

Figura 1. Familias de la codificación axial.

Fuente: elaboración propia.

Una vez realizada la codificación abierta, se procede a la construcción de códigos conceptuales, los cuales pertenecen a la fase relacional de la codificación axial. Se construyen tres grandes grupos de códigos; factores endógenos, factores exógenos y categorías emergentes. Cabe destacar que todos los grupos de códigos estuvieron presentes en cada uno de los cuatro focos de discusión. Se organizaron de acuerdo con el fundamentado que reflejaba Atlas-ti, cuyo resultado se presenta en la tabla 3. 
La excelencia académica de mujeres con discapacidad visual en la Universidad: aportes de la teoría fundamentada | María Dolores Yerga-Míguez, Marcela Ramírez-Morera y Rosa María Díaz-Jiménez

Tabla 3. Códigos conceptuales

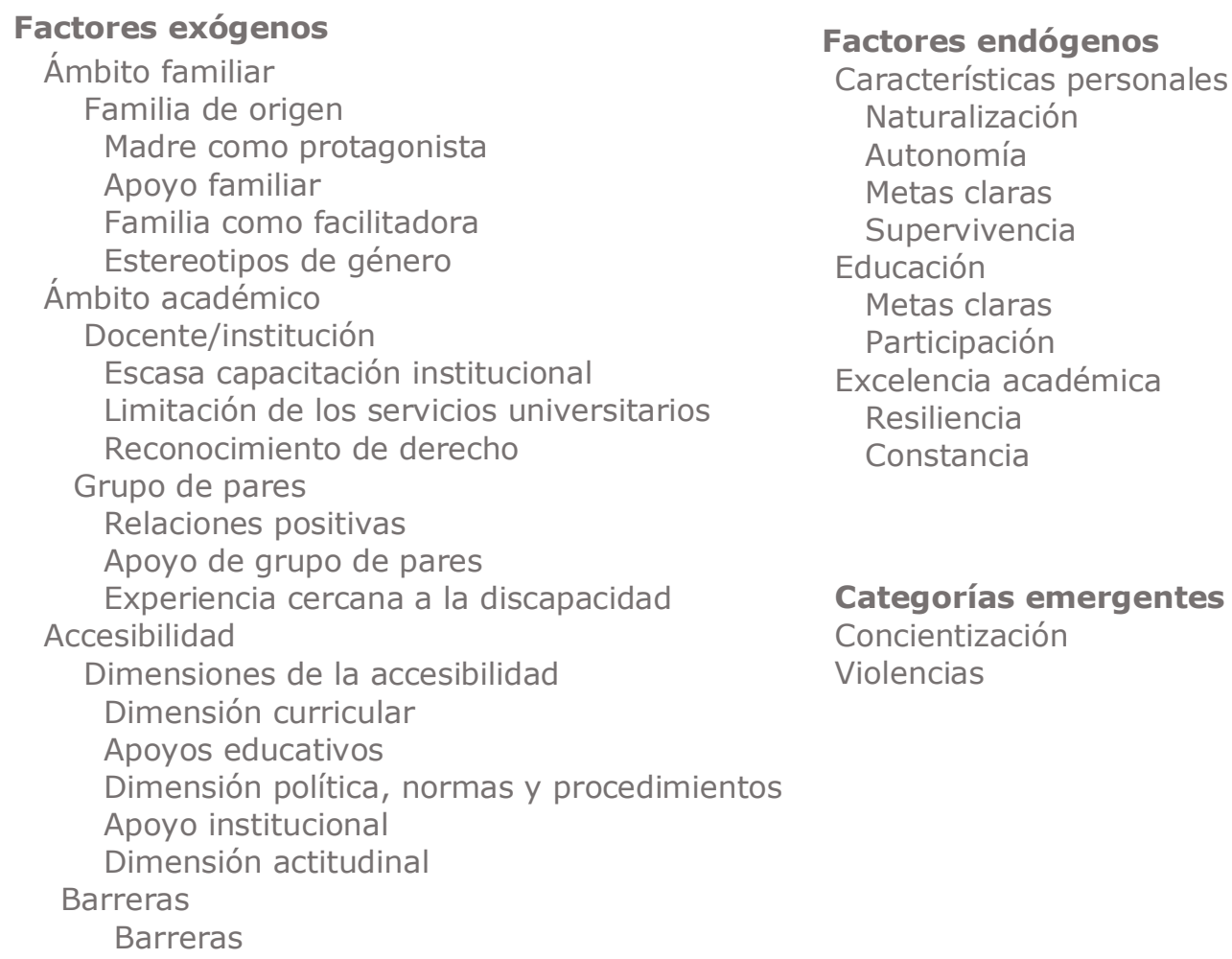

Fuente: elaboración propia a partir de UHA.

Una vez finalizada la codificación axial con los códigos conceptuales se procede a su interpretación. Pasando así a la codificación selectiva que se realiza a través de los tres códigos conceptuales de primer nivel.

\section{Resultados}

Los factores exógenos aglutinan los discursos del ámbito familiar e institucional. En este último se acogen los testimonios del grupo de pares y los docentes. En lo que a los factores endógenos respecta se analizan las características personales, sus emociones y percepciones, además de una aproximación al concepto de excelencia académica.

\section{Factores exógenos de influencia}

La familia emerge como un factor determinante en el itinerario académico de las mdv, según las personas entrevistadas por su implicación, apoyo permanente e impacto de la discapacidad en el desarrollo del propio contexto familiar. En la tabla 4 se presenta una triangulación de los discursos sobre implicación del ámbito familiar. 
La excelencia académica de mujeres con discapacidad visual en la Universidad: aportes de la teoría fundamentada | María Dolores Yerga-Míguez, Marcela Ramírez-Morera y Rosa María Díaz-Jiménez

Tabla 4. Discursos sobre la implicación familiar

\begin{tabular}{|c|c|c|c|}
\hline Ámbito & $\begin{array}{l}\text { Código } \\
\text { común }\end{array}$ & Mujer española (C1P1) ${ }^{1}$ & $\begin{array}{ll}\text { Mujer } & \text { costarricense } \\
(\mathrm{C} 2 \mathrm{P} 2) & \end{array}$ \\
\hline \multirow{3}{*}{ Familiar } & $\begin{array}{l}\text { Implicación } \\
\text { de la familia }\end{array}$ & $\begin{array}{l}\text { "También tuve la suerte que mi } \\
\text { madre, con ocho años, se empeñó } \\
\text { que aprendiera mecanografía, que es } \\
\text { lo que a día de hoy me ha salvado la } \\
\text { vida" (E7C1P1:100). }\end{array}$ & $\begin{array}{l}\text { "Estudié en Pérez } \\
\text { Zeledón, [...] pero mis } \\
\text { papás, por la condición de } \\
\text { discapacidad, me trajeron } \\
\text { para San José, porque les } \\
\text { dijeron que había mejores } \\
\text { opciones acá" (E4C2P2:14). }\end{array}$ \\
\hline & $\begin{array}{l}\text { Desarrollo } \\
\text { adaptativo } \\
\text { de la familia }\end{array}$ & $\begin{array}{l}\text { "Yo me daba cuenta de que ella } \\
\text { miraba como de reojo y entonces yo } \\
\text { la empecé a llevar a médicos [...] } \\
\text { hasta que ya llegamos a un médico } \\
\text { que me dijo que creía saber lo que } \\
\text { tenía. [...], vamos yo seguí viendo } \\
\text { médicos para ver si este hombre se } \\
\text { había equivocado o no y me } \\
\text { confirmaron" (E6C1F1:6). } \\
\text { "El padre [...] se quitaba del medio, } \\
\text { no ha sabido afrontar los problemas y } \\
\text { de hecho no sabemos nada" } \\
\text { (E6C1F1:28). }\end{array}$ & $\begin{array}{l}\text { "Tal vez sí pensaba que } \\
\text { iba a tener que ser el apoyo } \\
\text { de ella, o sea, que yo iba a } \\
\text { tener que ayudarla en todo y } \\
\text { que en muchas cosas ella tal } \\
\text { vez iba a ser una carga, } \\
\text { porque siempre iba a tener } \\
\text { que estarla halando a todas } \\
\text { partes" (E2C2F2:15). }\end{array}$ \\
\hline & $\begin{array}{l}\text { Apoyo } \\
\text { permanente }\end{array}$ & $\begin{array}{l}\text { "En mi casa miraba con mi madre } \\
\text { el plano dónde está el aula para poder } \\
\text { llegar" (E7C1P1:47). }\end{array}$ & $\begin{array}{l}\text { "La he apoyado mucho y } \\
\text { le he dicho que mientras ella } \\
\text { quiera estudiar puede } \\
\text { hacerlo" (E2C2F2:21). }\end{array}$ \\
\hline
\end{tabular}

Fuente: elaboración propia a través de la UHA.

El papel protagonista de la madre es fundamental para las mujeres entrevistadas, ha sido un impulso de gran relevancia para la excelencia académica en el caso español. También el apoyo familiar (Díaz, 2003), la comunicación y diálogo constante y la experiencia cercana a la discapacidad, convierten a este grupo primario en facilitador dentro de los procesos educativos brindando apoyos constantes a las estudiantes, tal y como se observa en el contexto costarricense, donde la familia se ve abocada a cambiar su lugar de residencia en busca de un contexto escolar adaptado a las necesidades de la MDV. En ambos contextos se reconocen los gastos asociados a la discapacidad y la necesidad de contar con apoyos económicos para continuar los diversos procesos educativos.

Asimismo, dentro del proceso de ajuste a la discapacidad (Barrientos, 2017) muchas familias transitan por las distintas etapas que les dificulta la comprensión de diferentes situaciones asociadas a la discapacidad, por ejemplo, experimentan las fases de shock y embotellamiento, la de anhelo y búsqueda de la figura perdida, así lo manifiesta la madre de la mujer española. Al proceso de discapacidad se le suma el abandono o distanciamiento en

Para identificar las citas de cada discurso ver tabla 2. Para identificar las citas textuales de las entrevistas se sigue el formato de Atlas.ti v. 8 "E-n"(siendo Eel n. ${ }^{0}$ de la entrevista y $n$ el n. ${ }^{0}$ de cita) seguido de la denominación de la unidad de observación. 
La excelencia académica de mujeres con discapacidad visual en la Universidad: aportes de la teoría fundamentada | María Dolores Yerga-Míguez,

Marcela Ramírez-Morera y Rosa María Díaz-Jiménez

la cotidianeidad familiar por parte del padre. En cuanto a la pareja de la MDV costarricense se reconoce la incertidumbre en el comienzo de la relación.

En los diferentes análisis del discurso se evidencia que existe un esfuerzo permanente y constante de la familia. La MDV española explica cómo estudiaba el mapa de la universidad con la madre para luego poder llegar a las clases. Por su parte, la pareja de la MDV costarricense afirma que le apoya hoy día para que ella siga estudiando.

Entre otras cuestiones, aún prevalecen estereotipos vinculados con el género y sobre todo con la idealización de la maternidad, tanto en las MDV participantes como en la madre española. En el caso de la mujer costarricense, afirma que ella debe cumplir lo que está establecido socialmente para las madres con el objetivo de que su hija crezca con bienestar. No obstante, la madre española evidencia alcanzar mérito propio a través de los logros académicos desarrollados por su hija.

Además, es necesario mencionar inestabilidad laboral del entorno familiar, particularmente a la madre española, que apoya a la MDV con funciones de transporte, por ejemplo, y expone la dificultad de conciliar y la falta de apoyos institucionales que brinden recursos sociales, económicos y profesionales, entre otros.

\section{Interacciones en el ámbito académico}

Otros factores de influencia relevantes para las personas participantes se refieren a las interacciones en el ámbito académico que toman forma en las relaciones con los y las docentes y con los y las compañeras de la universidad. En ambos casos, la cuestión de relevancia está en la accesibilidad.

Con el grupo de pares se establece una relación positiva a través de interacciones que promueven la autonomía y contribuyen a su construcción identitaria. Se destaca la experiencia previa con personas con discapacidad de los y las compañeras de universidad, que han contribuido a una mayor comprensión y predisposición al acompañamiento de la MDV. Se percibe una escasa percepción de las posibles limitaciones y se naturaliza la condición de discapacidad de las mujeres.

En el diálogo de ambos docentes se perciben personalidades emprendedoras y con características de lideresa, de ambas mujeres. Además, se percibe en su desarrollo discursivo códigos como el previo contacto con personas con discapacidad que los han llevado a contar con una concienciación ante estas alumnas.

Existen códigos teóricos significativos originados en el proceso de codificación abierta y axial que se refieren a la escasa capacitación institucional, limitación de los servicios universitarios y el reconocimiento de la educación superior como derecho.

La escasa capacitación institucional se vincula con la dimensión de la accesibilidad actitudinal del profesorado (Holst, 2006; Ramírez, 2011), el cual requiere acceder a la información y a la interacción directa con el estudiantado en condición de discapacidad para la configuración de un conocimiento que contribuya a la comprensión de la realidad de la discapacidad bajo un paradigma social con el objetivo de evitar la reproducción de estereotipos y mitos en esta área que restringen el reconocimiento de la educación superior como derecho de las mujeres con discapacidad.

Es necesario reconocer la existencia de políticas internacionales que retoma el papel protagónico de las MDV y el derecho a la educación de esta población. Dichas normativas deben retomarse en las políticas públicas de los diferentes países, con el objetivo de aplicarlos en los lineamientos institucionales, particularmente, en los contextos universitarios mediante programas dirigidos a la capacitación y el desarrollo de los servicios de apoyo para el 
La excelencia académica de mujeres con discapacidad visual en la Universidad: aportes de la teoría fundamentada | María Dolores Yerga-Míguez, Marcela Ramírez-Morera y Rosa María Díaz-Jiménez

estudiantado en condición de discapacidad. Esta realidad es visible en el diálogo discursivo entre las mujeres participantes del estudio presentado en la tabla 5.

Tabla 5. La accesibilidad como meta común

\begin{tabular}{|c|c|c|}
\hline Ámbito & Mujer española (C1P1) & Mujer costarricense (C2P2) \\
\hline $\begin{array}{l}\text { Académico } \\
\text { (Accesibilidad) }\end{array}$ & $\begin{array}{l}\text { "Llega el curso y los profesores } \\
\text { ya estaban informados por el } \\
\text { director incluso de que me } \\
\text { habían hecho la prueba que yo } \\
\text { trabajaba así y yo el primer día } \\
\text { y me empiezan a ofrecer todo el } \\
\text { material ya en pendrive, en } \\
\text { Word, entonces yo, vamos en } \\
\text { mi vida me he encontrado ni me } \\
\text { volveré a encontrar } \\
\text { probablemente una disposición } \\
\text { tan buena" (E7C1P1:113). }\end{array}$ & $\begin{array}{l}\text { "Como yo fui coordinador de la } \\
\text { Comisión de Informática también } \\
\text { tenemos muy pendiente lo que era } \\
\text { el software que tenía que tener el } \\
\text { equipo con estos compañeros y nos } \\
\text { reuníamos justamente para eso" } \\
\text { (E3C2D2:40). } \\
\text { "El apoyo que yo creo que fue } \\
\text { esencial, muchas veces fue el } \\
\text { apoyo de otras personas a } \\
\text { facilitarle el proceso de enseñanza, } \\
\text { como la parte de la lectura o que el } \\
\text { docente leyera lo que estaba en la } \\
\text { pizarra o el material que estaba } \\
\text { exponiendo" (E4C2P2:22) }\end{array}$ \\
\hline
\end{tabular}

Fuente: Elaboración propia a partir de la UHA.

La accesibilidad es la posibilidad que tienen todas las personas, sin importar su condición, de ser partícipe en los diferentes ámbitos sociales, garantizando su seguridad, bienestar, movilidad, comunicación y realización holística (Ramírez, 2011). Se destacan la importancia de las categorías de apoyos educativos, institucionales y reconocimiento de derechos, las cuales se encuentran relacionadas con las dimensiones de la accesibilidad, curricular y administrativa (Ramírez, 2011).

La dimensión curricular se refiere a aquellos apoyos educativos presentes en los distintos procesos académicos desarrollados con las personas con discapacidad en el transitar de la vida universitaria. Además, se retoma aspectos relacionados con el área tecnológica y la accesibilidad en la comunicación vinculado con la actitud docente, factores importantes en las diferentes trayectorias académicas de las MDV.

La accesibilidad en todas las dimensiones ha estado presente en la totalidad del trayecto académico de ambas mujeres interactuantes de la investigación, factor fundamental para alcanzar la excelencia académica, particularmente en la accesibilidad actitudinal interactúan los apoyos educativos y la implicación del personal docente en su práctica profesional, la información y la capacitación en la discapacidad contribuyen a la configuración de conocimiento que permite la generación de espacios accesibles proporcionando el desarrollo constante de experiencias exitosas en los contextos universitarios.

Otra dimensión de la accesibilidad que resalta en el análisis discursivo presente en la investigación es la ambivalencia entre la percepción y los recursos de los servicios de apoyos, los cuales se refieren a las medidas, acciones y proyectos realizados desde la institucionalidad universitaria para garantizar el acceso, a permanencia y la finalización de los estudios universitarios de las personas con discapacidad, manteniendo la autonomía y su independencia (Ramírez, 2011). 
La excelencia académica de mujeres con discapacidad visual en la Universidad: aportes de la teoría fundamentada | María Dolores Yerga-Míguez,

Marcela Ramírez-Morera y Rosa María Díaz-Jiménez

En algunos momentos, los diálogos discursivos se inclinan por el reconocimiento efectivo de estos servicios de apoyos, mediante la facilitación de recursos que contribuyan a continuar en la vida académica.

Otro código relevante son los apoyos técnicos, los usos de dispositivos tecnológicos para la lectura audible, el uso de bastón, movilidad, ampliación de la letra, algunas especificaciones en espacios físicos como la iluminación y ubicarse cerca de la pizarra, incluso el acompañamiento del grupo de pares en algunas actividades académicas.

Tabla 6. Las barreras, una realidad transversal

\begin{tabular}{lll}
\hline Ámbito & Mujer española (C1P1) & Mujer costarricense (C2P2) \\
\hline Académico & "También creo que la universidad & "[...] La parte de infraestructura \\
(Barreras) & $\begin{array}{l}\text { adquiere un compromiso de apoyar } \\
\text { a la igualdad de oportunidades, que }\end{array}$ & sobre todo el edificio de Ciencias \\
& estas personas no tienen igualdad & Sociales, en ese momento solo \\
& de oportunidades, es que les cuesta & estaba el ascensor y se iba la luz \\
& la asignatura les cuesta muchísimo & mucho antes y a las personas con \\
& más, muchísimo más que a otra discapacidad les costaba mucho \\
& cualquiera, porque no tiene un & subir y las clases a lo mejor eran en \\
apoyo real" (E5C1D1:53). & el cuarto piso" (E4C2P2:36).
\end{tabular}

Fuente: elaboración propia a través de la UHA.

Junto a los discursos sobre la accesibilidad emerge el cuestionamiento de las barreras con mayor frecuencia de código. La carencia de apoyo institucional, limitación en la inversión económica en los servicios de apoyo y en la infraestructura universitaria, así como las barreras actitudinales por parte del profesorado constituyen esta categoría. Además de las barreras físicas, aún existentes en los contextos universitarios. Los productos de apoyo tecnológico continúan generando algunos tipos de limitaciones en su uso, pueden limitar el curso de procesos académicos y restringir la autonomía e independencia de las mujeres con discapacidad.

Las barreras actitudinales (Ramírez, 2011) persisten en los diferentes contextos educativos, las cuales se constituyen en acciones que restringen el desarrollo autónomo. Por lo tanto, las MDV en este diálogo discursivo enfatizan en la existencia de la discriminación y falta de empatía por parte del profesorado.

Del análisis de los diálogos se extrae que los sistemas educativos universitarios vienen intentando desarrollar acciones que contribuyan a la inclusión de las personas con discapacidad, dentro de su accionar institucional. Sin embargo, los intentos han sido limitados y aún existe la necesidad de avance para el reconocimiento de la educación superior como derecho.

De estas carencias surgen iniciativas que propician espacios para apoyar a las personas con discapacidad en los ámbitos universitarios. Por ejemplo, el establecimiento de servicios de apoyos que persiguen establecer contactos entre el cuerpo docente y el estudiantado para asesorar y brindar apoyos educativos a aquellas MDV que desean culminar su carrera universitaria con excelencia académica.

A pesar de la ausencia de recursos económicos institucionales específicos en discapacidad, se destacan apoyos económicos vinculados con la excelencia académica que fortalecen y permiten la culminación de una carrera universitaria. Una muestra de ello se observa en los diálogos de las MDV. 
La excelencia académica de mujeres con discapacidad visual en la Universidad: aportes de la teoría fundamentada | María Dolores Yerga-Míguez, Marcela Ramírez-Morera y Rosa María Díaz-Jiménez

Las experiencias académicas de las MDV han coexistido entre distintas realidades, por ejemplo, han recibido apoyo de algún profesorado y grupos de pares. También han tenido que derribar barreras de accesibilidad, ya sean actitudinales, físicas, tecnológicas, administrativas y normativas, entre otras. Aun así, ellas continuaron su trayecto académico hasta alcanzar la excelencia académica, donde su motor principal está relacionado con sus características endógenas.

\section{Factores endógenos de influencia}

Los diálogos sobre los factores endógenos describen algunas características personales de las mdv, sus emociones, la percepción de metas claras y firmeza en conseguir objetivos y la propia excelencia académica que se presupone.

Tabla 7. Diálogo entre las características personales de las MVD

\begin{tabular}{lll}
\hline Ámbito & Mujer española (C1P1) & Mujer costarricense (C2P2) \\
\hline Personal & "Y entonces, yo me acuerdo que me & "Creo que mi familia ha sido como una \\
trataban con demasiada admiración & columna ahí, detrás mío, que me \\
& {$[\ldots]$ la mayoría de las veces yo incluso } & enseñó que mi condición era parte de \\
tenía que frenar un poco un exceso de & mí y que eso no me tenía que limitar \\
reconocimiento $[. .$.$] y yo decía j*** no$ & para alcanzar lo que yo me proponía \\
& me admires porque yo apruebo y & {$[\ldots]$ mi familia no fue sobreprotectora, } \\
& suspendo como todo el mundo y tengo & me enseñó a ser muy independiente \\
& que estudiar y tengo mala leche y & {$[\ldots . .$.$] " (E4C2P2:65).$} \\
& tengo tal" (E7C1P1:44).
\end{tabular}

Fuente: elaboración propia a través de la UHA.

La naturalización de la condición de discapacidad es una característica personal que resalta en las dos mujeres protagonistas, tal y como se muestra en la tabla anterior y que refieren otros actores del análisis. La autonomía ha sido promovida por el apoyo familiar y el propio interés de la mujer por desenvolverse en su entorno y las metas claras, aunque han existido momentos de incertidumbre o dudas, se han resuelto por el interés hacia el aprendizaje de la mujer.

Una característica común en ambas mujeres es lo que describen como el espíritu de supervivencia. Ambas mujeres manifiestan tener una autoimagen positiva. Por otro lado, ambas han sido alumnas que han participado de la vida cultural y universitaria.

Con respecto a la perseverancia, aunque supone también un elemento descriptor de la excelencia académica, se categoriza como constructor identitario de las mujeres protagonistas. La independencia se entiende como la capacidad de realizar tareas por sí mismas y la autonomía, como aquella posibilidad de elegir qué tareas hacer bajo su propio criterio. La sensibilidad a la justicia es otra característica relevante de ambas mujeres, aunque puede vincularse a la condición de discapacidad o a su profesión.

Finalmente, entre los códigos de menor fundamentación, y como pautas comunes en ambos contextos, se destacan la adaptación de la mujer, su forma decisiva, las habilidades sociales, el tipo de condición de discapacidad, la confianza, ser inquietas, ser activistas, reflexivas, buscadoras de soluciones, socializadoras y comprometidas. En igual medida, aparece la 
La excelencia académica de mujeres con discapacidad visual en la Universidad: aportes de la teoría fundamentada | María Dolores Yerga-Míguez, Marcela Ramírez-Morera y Rosa María Díaz-Jiménez

desmotivación y el emprendimiento, porque ambas viven momentos decisivos y de crecimiento personal. La necesidad de aprendizaje es un elemento intrínseco en todo el discurso de las MDV, al igual que la relación con el entorno y su autoestima.

A través del discurso de las MDV y su entorno, se destacan emociones de incertidumbre, relacionadas con una sensación de necesaria supervivencia durante todo el proceso académico. A su vez, emergen testimonios de satisfacción por la culminación de objetivos, ya que la educación superior se tornaba inalcanzable en algunos momentos de incertidumbre.

Desde la perspectiva de la autodeterminación, el código protagonista es "tener metas claras" y tiene que ver con la firmeza de las mujeres, la toma de decisiones y el carácter crítico en el desarrollo de sus carreras académicas. A partir de la UHA, se evidencia que la estudiante costarricense tiene autonomía y decisión, sabe lo que quiere hacer y hasta dónde ir. Habla en primera persona y aclara que sus padres les explicaron las limitaciones económicas, pero siempre mostraron apoyo incondicional a sus elecciones. En el caso español, "metas claras" tiene el sentido de satisfacción de las expectativas académicas.

La valoración de los apoyos educativos y el aprendizaje en el proceso académico son códigos que se relacionan con la educación, pero también con las características personales. Con menor densidad encontramos la continuidad en los estudios, la importancia de entender la tarea, la movilización social y la exigencia académica.

\section{Un camino necesario: la concientización}

En los discursos analizados, la concientización hace referencia a la transformación que se produce en los distintos contextos de las MDV y cuando las otras personas exploran y conocen el mundo de la discapacidad. En este proceso de transformación, tiene diversos grados, desde la escasa hasta la alta concientización. Cuando la concientización es alta hablamos de naturalización de la diversidad funcional. En este caso la compañera de Costa Rica muestra una escasa concientización: "A nivel personal sí tienen habilidades, pero les falta más información porque [sic] ellos tienen toda la buena actitud" (E1C2C2:20).

Hasta llegar a la concientización que permite la naturalización, como expresa el docente del caso español: "La diversidad no es una excusa, es una situación y hay que hacer que la persona no tenga que adaptarse a las cosas, sino que las cosas se adapten a la persona, esa es nuestra obligación" (E5C1D1:52).

Se reconoce así la concientización, según lo planteado por Freire en palabras de Kirkwood y Kirkwood:

Proceso mediante el que las personas son estimuladas y animadas a explorar su realidad y su conciencia, de forma que la comprensión de la realidad y de su propia conciencia es cada vez más profunda, y comienza a comprometerse como praxis. (2011, p. 172)

Cuando se trabaja con el término naturalización se tiende a dar por supuesto que existe un antagónico. Así, la naturalización se expresa en este estudio como el trato igualitario a las personas con o sin discapacidad. 
La excelencia académica de mujeres con discapacidad visual en la Universidad: aportes de la teoría fundamentada | María Dolores Yerga-Míguez, Marcela Ramírez-Morera y Rosa María Díaz-Jiménez

\section{Violencias}

Las situaciones a las que en las que se ha visto expuesta la MDV española hacen necesaria la codificación de la violencia.

Tabla 8. Testimonio de violencias

\begin{tabular}{|c|c|}
\hline $\begin{array}{l}\text { Código } \\
\text { emergente }\end{array}$ & Mujer española (C1P1) \\
\hline Violencias & $\begin{array}{l}\text { "Hago una prueba de acceso en un instituto } \\
\text { [...], donde me encuentro con un director que me } \\
\text { dice que él no tiene por qué hacerme la adaptación } \\
\text { que yo decía que él me hará la que él considere. } \\
\text { Llegó el día de la prueba de acceso y me ha hecho, } \\
\text { me da un examen completamente normal y me } \\
\text { dice que si yo no puedo hacer examen, pues que } \\
\text { a lo mejor tampoco puedo seguir estudiando" } \\
\text { (E7C1P1:53). } \\
\text { "Me he visto en situaciones muy dantescas. } \\
\text { [...] pese a que me revisaron, bueno esta mujer } \\
\text { pidió que el portátil se quedará una semana en la } \\
\text { oficina para que yo no le metiera nada, a ver si yo } \\
\text { lo necesito para estudiar que aparte de mi } \\
\text { herramienta examen es también mi herramienta } \\
\text { de trabajo [...] cuando llegué al examen me colocó } \\
\text { a cada lado de las bancas libres a una persona } \\
\text { vigilante" (E7C1P1:65). }\end{array}$ \\
\hline
\end{tabular}

Fuente: elaboración propia a través de la UHA.

En primer lugar, siguiendo su propio testimonio, la intimidación y cuestionamiento por parte del profesorado y la dirección del centro podría ser considerada por Rodríguez (2010) como violencia escolar de tipo psicológico.

Se desarrolla una exposición de la discapacidad desde el paradigma tradicional, por parte, especialmente, de la pareja de la mujer costarricense, además de observarse a lo largo de todas las unidades de observación. Un paradigma en el que el paternalismo limita la capacidad de actuación libre de las personas con discapacidad, más aún si cabe de las mujeres. Es por eso por lo que esta línea argumental también se acoge a las posibles violencias que han podido incurrir en las MDV estudiadas. Cabe destacar que, en el caso de la MDV de Costa Rica, no emite ningún acontecimiento de este tipo, posiblemente no porque no lo haya vivido, sino porque no tiene las herramientas necesarias para asumirlas y verbalizarlas, como sí ocurre en el caso de la mujer española. 
La excelencia académica de mujeres con discapacidad visual en la Universidad: aportes de la teoría fundamentada | María Dolores Yerga-Míguez, Marcela Ramírez-Morera y Rosa María Díaz-Jiménez

\section{Discusión}

Los resultados obtenidos en esta investigación reafirman la relación de la excelencia académica, del éxito de las MDV en su itinerario universitario con características resilientes. Se entiende por resiliencia "un proceso dinámico que puede ser impulsado en cualquier organismo al estar expuesto a la adversidad y el estrés generado por ésta" (Aguilar-Maldonado et al., 2019, p. 77). Esta definición coincide con lo estudiado por Dueñas et al. (2019), quienes analizan cómo, desde la Organización para la Cooperación y el Desarrollo Económicos (OCDE), se utiliza la resiliencia para distinguir al alumnado que destaca académicamente, a pesar de vivir en un entorno de nivel socioeconómico bajo. Asimismo sucede con las MDV, debido que ellas se encuentran constantemente derribando barreas impuestas por un contexto discapacitante, generador de las condiciones de discapacidad.

En cuanto a las características necesarias para el finalizar con éxito los estudios, expuestas por Cabrera et al. (2006), los resultados reafirman rasgos como la capacidad de esfuerzo y superación, motivación, metas claras a largo plazo y constancia, actitudes que se han visto reflejadas en ambos contextos. En relación con la capacidad de esfuerzo y superación, aspecto reseñable en ambos contextos, hay que realizar un cuestionamiento sobre el campo de batalla en el que se convierte, en muchas ocasiones, el itinerario académico para las MDV. Por otro lado, la constancia es una característica definitoria de ambas mujeres, al igual que el sentimiento de realización personal, que, si bien es subjetivo, evidencia la consecución de los objetivos. Además, también se ve reflejado en ambos contextos la motivación hacia el aprendizaje a través de los testimonios de las compañeras de las MDV.

Según los discursos analizados, la excelencia académica también necesita de capacidad crítica. Se destaca que la excelencia académica no tiene una vinculación directa con la calificación y tampoco con las cotas académicas, la describen según las metas personales y el desarrollo de la vida académica de la mujer, además de otras características como la perseverancia, las ideas propias, carácter de lideresa, mujeres con mucha cultura, impertinentes, luchadoras, proactivas y rebeldes.

\section{Conclusiones}

Este trabajo se ha planteado identificar factores de influencia en itinerarios académicos de MDV en estudios superiores, conocer apoyos recibidos por estudiantes universitarias con discapacidad visual y comparar contextos de influencia en las trayectorias académicas de MDV.

Como factores de influencia vinculados con la excelencia académica o el éxito percibido por las propias mujeres en sus itinerarios académicos, se identifican como factores exógenos la familia, el grupo de iguales (compañeros/as) y el equipo docente como apoyos permanentes. Sin embargo, la familia influye de manera ambivalente, a veces como el apoyo más importante y otras veces como barrera, como limitación de la participación de las MDV en la vida universitaria y en la autonomía para tomar decisiones.

Con respecto al entorno académico, se observa una relación positiva con compañeros y compañeras de estudios y también con el profesorado estudiado, aunque los discursos muestran déficit de concienciación en estos colectivos. El profesorado y el alumnado apunta a que la concienciación ampliaría opciones de accesibilidad en el ámbito universitario. El entorno académico sigue mostrando importantes barreras que hacen que las MDV se sientan invitadas 
graciables de la universidad, es decir, personas dependientes de la buena voluntad del profesorado y el resto del alumnado y no tanto estudiantes con plenos derechos.

Por su parte, los factores endógenos que favorecen la excelencia académica se relacionan con el esfuerzo, desempeño y actitud individual, cuyo eje principal queda constituido por las características resilientes desarrolladas. Las MDV son perseverantes y antes que estudiantes universitarias se muestran como combatientes de un sistema educativo con diversas formas de violencia.

Los apoyos recibidos por parte de su familia han sido principalmente de aliento para la persistencia en el itinerario académico. En cuanto a los apoyos por parte de sus iguales, la naturalización y el trato igualitario han supuesto la inclusión de las MDV en la vida universitaria. Por último, en lo que a los docentes se refiere, las adaptaciones de materiales y el cuestionamiento ante las barreras actitudinales han servido de empuje para el aprendizaje.

Finalmente, ambos casos destacan en capacidad resolutiva, la claridad en la elección de metas y objetivos y constancia en el desempeño. Aunque la violencia ha sido un hallazgo del análisis, en uno de los casos no hay conciencia de ella, lo que hace pensar en la generación de herramientas que permitan la concienciación respecto a las violencias contra personas con discapacidad en los estudios superiores.

A pesar de la limitación de los casos analizados, lo representativo de los discursos permite avanzar en las dimensiones analizadas en este trabajo. Visibilizar itinerarios académicos de MDV de éxito aporta elementos de concienciación. Dar relevancia al impacto de los ámbitos familiares e institucionales en las trayectorias universitarias de MDV es un aporte necesario para avances en la inclusión educativa en las universidades.

Finalmente, se abre una vía de estudio sobre las violencias que se ejercen contra las mujeres con discapacidad en el ámbito académico.

\section{Referencias}

Aguilar-Maldonado, S., Gallegos-Cari, A. y Muñoz-Sánchez, S. (2019). Análisis de componentes y definición del concepto resiliencia: una revisión narrativa. Revista de Investigación Psicológica, (22), 77-100.

Aquino, S., Izquierdo, J., García, V. y Valdés, Á. (2016). Percepción de estudiantes con discapacidad visual sobre sus competencias digitales en una universidad pública del sureste de México. Apertura (Guadalajara, Jal.), 8(1), 8-9.

Araújo, C., Paz-Lourido, B. y Gelabert, S. (2016). Tipos de apoyo a las familias con hijos con discapacidad y su influencia en la calidad de vida familiar. Ciência \& Saúde Coletiva, 21, 3121-3130.

Barbosa, J. (2019). Uso de técnicas de lectura fácil como método para el acceso al currículo de un alumno con sordoceguera. Integración: Revista Digital sobre Discapacidad Visual 75, 125-133. https://www.once.es/dejanos-ayudarte/la-discapacidad-visual/revistaintegracion/2019-integracion-74-75/numero-75/barbosa-uso-de-tecnicas-de-lectura-facilcomo-metodo-para-el-acceso-al-curriculo/download 
La excelencia académica de mujeres con discapacidad visual en la Universidad: aportes de la teoría fundamentada | María Dolores Yerga-Míguez, Marcela Ramírez-Morera y Rosa María Díaz-Jiménez

Barrientos, P. (2017). La participación familia-escuela en México desde un enfoque inclusivo: reflexiones sobre familia y discapacidad. Revista Latinoamericana de Educación Inclusiva, 7(2), 175-193.

Bonilla-Castro, E. y Sehk, P. (2005). Más allá del dilema de los métodos: la investigación en ciencias sociales. Editorial Norma.

Cabrera, L., Bethencourt, J., Álvarez, P. y González, M. (2006). El problema del abandono de los estudios universitarios. Relive, 171-203. https://doi.org/10.7203/relieve.12.2.4226

Coller, X. (2005). Estudio de casos. Centro de Investigaciones Sociológicas (CIS).

Consejo Nacional de Rectores. (2017). Estado de la educación costarricense. https://www.estadonacion.or.cr/educacion2017/assets/ee6-informe-completo.pdf

Corona, A. y Díaz-Jiménez, R. (2016). La participación de las mujeres con diversidad funcional física en el sur de España: un análisis desde la interseccionalidad. Centro de Estudios Políticos y Constitucionales.

Díaz, R. (2003). Personas con discapacidad. Una aproximación desde el trabajo social. Aconcagua Libros.

Díaz, R. y Serrato, M. (2016, noviembre). La conquista de la excelencia de las mujeres con discapacidad en la universidad. En Actas del XV Congreso Nacional de Educación Comparada: Ciudadanía Mundial y Educación para el Desarrollo. Una mirada Internacional. Sociedad Española de Educación Comparada y Universidad Pablo de Olavide, Sevilla.

Dueñas, X., Godoy, S., Duarte, J. y López, D. (2019). La resiliencia en el logro educativo de los estudiantes colombianos. Revista Colombiana de Educación, 76, 69-90.

Fundación Universia. (2019, 9 de mayo). Estudio sobre el grado de inclusión del sistema universitario español respecto de la realidad de la discapacidad (IV). https://www.fundacionuniversia.net/content/dam/fundacionuniversia/pdf/estudios/IVEstu dio_UniversidadyDiscapacidad_ACC.pdf

Glaser, B. y Strauss, A. (1967). The discovery of grounded theory. Aldine Press.

Gobierno de España. (2013). Real Decreto Legislativo 1/2013, de 29 de noviembre, por el que se aprueba el Texto Refundido de la Ley General de derechos de las personas con discapacidad y de su inclusión social. Boletín Oficial del Estado, 289 (I), 95635-95673. https://www.boe.es/boe/dias/2013/12/03/pdfs/BOE-A-2013-12632.pdf

Heiman, T. (2006). Social support networks, stress, sense of coherence and academic success of university students with learning disabilities. Social Psychology of Education, 9(4), 461478. https://doi.org/10.1007/s11218-006-9007-6

Holst, B. (2006). Estudio bibliográfico: ayudas técnicas, tecnologías de apoyo y accesibilidad. Universidad Nacional de Colombia.

Ibáñez, J. (1985). Análisis sociológico de textos y discursos. Revista Internacional de Sociología, 43(1), 119-162.

Instituto Nacional de Estadísticas y Censos de Costa Rica (INEC) y Consejo Nacional de Personas con Discapacidad (Conapdis). (2019). Encuesta Nacional sobre Discapacidad 2018. San José, Costa Rica. https://www.inec.cr/sites/default/files/documetos-bibliotecavirtual/reenadis2018.pdf 
La excelencia académica de mujeres con discapacidad visual en la Universidad: aportes de la teoría fundamentada | María Dolores Yerga-Míguez,

Marcela Ramírez-Morera y Rosa María Díaz-Jiménez

Karimi, F. (2012). Differential levels of student-related predictors of academic success in the university. The International Journal of Learning, 18(5), 285-306. https://doi.org/10.18848/1447-9494/CGP/v18i05/47591

Kirkwood, G. y Kirkwood, C. (2011). Living adult education. Freire and Scotland. Sense Publishers.

Levitt, H., Bamberg, M., Creswell, J., Frost, D., Josselson, R. y Suárez-Orozco, C. (2018). Journal article reporting standards for qualitative primary, qualitative meta-analytic, and mixed methods research in psychology: The APA Publications and Communications Board task force report. American Psychologist, 73(1), 26-46. http://dx.doi.org/10.1037/amp0000151

Molina, A. (2019). Aula de tecnología para alumnado de Educación Infantil (0 a 6 años) con discapacidad visual. Integración: Revista Digital sobre Discapacidad Visual, 75, 99-124. https://www.once.es/dejanos-ayudarte/la-discapacidad-visual/revista-integracion/2019integracion-74-75/numero-75/molina-aula-de-tecnologia-para-alumnado-de-educacioninfantil/download

Moreira, T. (2009). Factores endógenos y exógenos asociados al rendimiento en matemática: un análisis multinivel. Revista Educación, 33(2), 61-80.

Moriña, A. y Melero, N. (2016). Redes de apoyo sociales y académicas de estudiantes con discapacidad que contribuyen a su inclusión en la enseñanza superior. Revista Prisma Social, (16), 32-59. https://revistaprismasocial.es/article/view/1252

Novo, I., Muñoz, J. y Calvo, N. (2015). Los futuros docentes y su actitud hacia la inclusión de personas con discapacidad. Una perspectiva de género. Anales de psicología, 31(1), 155171. https://dx.doi.org/10.6018/analesps.31.1.163631

O'Connor, P. y O'Hagan, C. (2016). Excellence in university academic staff evaluation: A problematic reality? Studies in Higher Education, 41(11), 1943-1957. https://doi.org/10.1080/03075079.2014.1000292

Oravec, J. (2017). The manipulation of scholarly rating and measurement systems: Constructing excellence in an era of academic stardom. Teaching in Higher Education, 22(4), 423-436. http://dx.doi.org/10.1080/13562517.2017.1301909

Polo, M. y López, M. (2015). Transition to employment of university students with disabilities: factors for success. Universitas Psychologica, 15(2), 273-286. http://dx.doi.org/10.11144/Javeriana.upsy15-2.teus

Ramírez, M. (2011). Las dimensiones de accesibilidad en la Universidad de Costa Rica Sede Rodrigo Facio, un acercamiento desde las perspectivas de discapacidad y género. Reflexiones, 90(2), 71-88.

Rea, A., Acle, G., Ampudia, A. y García, M. (2014). Caracterización de los conocimientos de las madres sobre la discapacidad de sus hijos y su vínculo con la dinámica familiar. Acta Colombiana de Psicología, 17(1), 91-103. https://doi.org/10.14718/ACP.2014.17.1.10

Reed, M., Kennett, D., Lewis, T., Lund, E., Stallberg, C. y Newbold, I. (2009). The relative effects of university success courses and individualized interventions for students with learning disabilities. Higher Education Research \& Development, 28(4), 385-400. https://doi.org/10.1080/07294360903067013

Rodríguez, E. (2011, enero). Jóvenes y violencias en América Latina: aprendizajes destacados y desafíos a priorizar, para mejorar el impacto de las políticas públicas. En Foto 
Internacional Pensar el Futuro: la Prevención que México Necesita. Centro Nacional de Prevención del Delito y Participación Ciudadana, Montevideo.

Rodríguez, G. y Valenzuela, B. (2019). Acceso y permanencia de estudiantes con discapacidad en las universidades chilenas. Sinéctica, 53, 1-16.

Rodríguez, S. y Cano, A. (2015). Discapacidad y políticas públicas. Catarata.

Samudio, A. y Edda, O. (2016). El acceso de las mujeres a la educación superior. La presencia femenina en la Universidad de los Andes. Procesos Históricos. Revista de Historia y Ciencias Sociales, 29(1), 77-101.

Stake, R. (1995). The Art of Case Study Research. Sage Publications Ed.

Strauss, A., y Corbin, J. (2016). Bases de la investigación cualitativa: técnicas y procedimientos para desarrollar la teoría fundamentada. Universidad de Antioquia.

Taylor, S., y Bogdan, R. (1987). Introducción a los métodos cualitativos de investigación. Paidós.

Trotta, M., Lehtomäki, E. y Matonya, M. (2015). As capable as other students: tanzanian women with disabilities in higher education. International Journal of Disability, Development and Education, 62(2), 202-214. https://doi.org/10.1080/1034912X.2014.998178

Vallés, M. (1999). Técnicas cualitativas de investigación social. Reflexión metodológica e investigación social. Síntesis.

Villavicencio, C. y López, S. (2017). Presencia de la discapacidad intelectual en la familia, afrontamiento de las madres. Fides et Ratio-Revista de Difusión Cultural y Científica de la Universidad La Salle en Bolivia, 14(14), 99-112.

Yin, R. (1994). Discovering the future of the case study. Method in evaluation research. Evaluation Practice, 15(3), 283-290. 